\title{
Innovación educativa y producción de identidades: el caso del Programa Interdisciplinario de Investigación Escolar ${ }^{1}$
}

\author{
Rodrigo Mayorga ${ }^{2}$ \\ ORCID: 0000-0003-2995-5524 \\ Javier Pascual ${ }^{3}$ \\ ORCID: 0000-0001-6850-7391
}

\section{Resumen}

Las innovaciones educativas son procesos disruptivos dentro de los establecimientos educativos, y pueden tener efectos no sólo en los aprendizajes de los estudiantes, sino también en otros aspectos de la vida y la cultura escolar, incluida la identidad de sus distintos actores. El objetivo de este estudio es comprender cómo una innovación educativa puede abrir nuevos espacios de configuración de identidad en un establecimiento escolar y contribuir a la producción de significados por parte de los estudiantes y profesores involucrados. Para cumplir con dicho objetivo realizamos un estudio de caso de corte cualitativo, analizando el Programa Interdisciplinario de Investigación Escolar, un proyecto innovador de un establecimiento en Santiago de Chile en el que se pretende que estudiantes de educación secundaria formen competencias de investigación científica y académica. Aplicamos entrevistas semiestructuradas a los diversos actores involucrados en el proyecto, las cuales fueron analizadas siguiendo las líneas de la teoría fundamentada. Los resultados muestran que una innovación puede promover un espacio de producción de nuevas identidades a través de la emergencia de discursos, prácticas y reconocimiento externo, a la vez que impacta el autoconocimiento de los estudiantes y el desarrollo profesional de los profesores y genera un fuerte sentido de comunidad entre sus miembros, aunque bajo el riesgo de reproducir dinámicas de exclusión propias de la escuela.

\section{Palabras clave}

Construcción de identidad - Identidad profesional - Innovación educativa - Sentido de pertenencia - Producción del conocimiento.

1- Agradecemos al colegio Sagrados Corazones de Manquehue, que nos ha abierto sus puertas para conocer este proyecto y ha dado su autorización explícita para ser identificada en este estudio.

2- Columbia University, New York, Estados Unidos. Contacto: Irm2166@tc.columbia.edu

3- Universitat Autònoma de Barcelona, Barcelona, España. Contacto: jpascualmedina@gmail.com

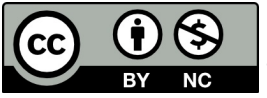




\section{Identity production and educational innovation: the case of the Interdisciplinary School Research Program}

\section{Abstract}

Educational innovations are disruptive processes within schools and can affect not only students' learning but also other aspects of their lives and of their schools' culture, such as the identity of its members. This study attempts to understand how an educational innovation opens up new spaces of identity configuration within a school and how students and teachers produce meanings by participating in it. To do so, we present a case study from the Interdisciplinary School Research Program about a school named Sagrados Corazones de Manquehue, based on semi-structured interviews with the actors involved in this project and an analytic approach drawing from grounded theory. Our findings show that innovation can configure a space in which new identities are produced through emergent discourses, practices, and external acknowledgement. At the same time, this new space impacts both the students' self-knowledge and the teachers' professional development, and furthers a strong sense of community among the members of the program. However, it also implies a constant risk of reproducing some of the excluding dynamics of the school.

\section{Keywords}

Identity production - Professional identity - Educational innovation - Belonging - Knowledge production.

\section{Introducción}

Los acelerados cambios que vive nuestra Sociedad del Conocimiento suponen un giro radical en cómo concebimos nuestro entorno. Hoy, creatividad e innovación ocupan un papel central, y el conocimiento se entiende como fuente principal del desarrollo económico y de la reestructuración de las relaciones sociales (SAHLBERG; B0CE, 2010; ASTUDILLO; IMBARACK, 2013). La escuela no queda ajena a esto, pues se espera mucho más de ella (RIVAS, 2010), a la vez que las fuentes de información externas al marco escolar cobran renovada importancia (JONNAERT et al., 2008). En este nuevo escenario, la innovación educativa se vuelve pieza clave, implicando no solo nuevos contenidos y metodologías, sino una reestructuración y reculturización de la escuela tradicional y el sistema escolar en su conjunto (FULLAN, 2007).

Innovar en su acepción más típica significa alterar algo introduciendo novedades, aunque en una definición más completa está asociada a la noción de cambio, entendido como una modificación que genera nuevas respuestas ante problemáticas tanto rutinarias como imprevistas (GAIRÍN; RODRÍGUEZ-GÓMEZ, 2011). Pero aunque la innovación siempre 
signifique cambio, no todo cambio es innovador. La literatura menciona tres criterios para poder hablar de innovación educativa: 1) debe estar intencionalmente orientado hacia el mejoramiento educativo desde su concepción (MURILLO, 2002); 2) pretende ser sostenible en el tiempo (ALTOPIEDI; LÓPEZ JIMÉNEZ, 2010); y 3) dicha sostenibilidad se da a través de un proceso crítico en que tanto el cambio como la misma institución se van adaptando ante limitaciones contextuales, institucionales y profesionales, así como por las resistencias y tensiones que surjan de actores de la comunidad escolar (MONTERO; GEWERC, 2010). Si bien la innovación puede ser entendida desde una mirada meramente técnocrática, estos supuestos también se condicen con una perspectiva sociocognitiva respecto al cambio, que lo concibe como resultado de una fuerte disonancia cognitiva entre la realidad percibida y el ideal de las personas, lo que lleva a procesos críticos que les incitan a modificar la realidad. De esta forma, toda innovación se compone de procesos, obstáculos y actores multifacéticos, interconectados y traslapados, construyendo su propio contexto en un proceso dinámico de aprendizaje individual y social (KEZAR, 2001).

Diversos estudios analizan la influencia de las estructuras y culturas escolares sobre los procesos de innovación educativa, o estudian como los actores educativos pueden incidir en dichos procesos (ALTOPIEDI; LÓPEZ JIMÉNEZ, 2010; ANDERSON, 2013; GAIRÍN; RODRÍGUEZ-GÓMEZ, 2011; MENCHÉN, 2012; SEPÚLVEDA; MURILLO, 2012). Escasa literatura ha seguido la dirección contraria, analizando el impacto de las innovaciones educativas sobre las culturas y los actores escolares más allá de los efectos instrumentales de ideas innovadoras analizadas con diseños experimentales. García (2005), por ejemplo, examina la interacción entre las innovaciones educativas y la cultura escolar manifestada en las relaciones de poder dentro de los centros escolares, descubriendo que a pesar de las resistencias para mantener el status quo, es la elaboración de objetivos comunes lo que permite aunar voluntades y abrir nuevas esperanzas de cambio. Esto se condice con las perspectivas del Interaccionismo Simbólico que plantean que es posible que los actores en cooperación construyan nuevos mundos simbólicos, a pesar de que las estructuras puedan ofrecer resistencias (LOPES, 2008). Todo esto sugiere que, además de incidir en los valores comunes de la institución, una innovación educativa puede incidir en las prácticas de los actores educativos y en sus estructuras valóricas y simbólicas.

Es importante recordar que los espacios escolares son sitios de reproducción (BOURDIEU; PASSERON, 2005) pero también escenarios de encuentros entre lo individual y lo institucional que generan “[...] espacios de producción, contingencia, creación e innovación” (REYES JUÁREZ, 2009, p. 152). A través de los recursos a los que tienen acceso en estos espacios y por medio de prácticas y artefactos culturales que utilizan en la producción de sus propias identidades, los miembros de una comunidad escolar pueden apropiarse de éstos y resignificarlos. Por ello resulta fundamental comprender cómo las instituciones y sus procesos de innovación pueden contribuir a la producción de nuevas prácticas y artefactos culturales que permitan esta constante resignificación. Nuestro estudio analiza de forma profunda una innovación educativa particular -el Programa Interdisciplinario de Investigación Escolar del colegio Sagrados Corazones de Manquehue-, con el objetivo de comprender de qué manera abre nuevos espacios de configuración de identidad en un establecimiento escolar y contribuye a la producción de significados por parte de los actores involucrados en ella. 


\section{Marco teórico}

Identidad es un concepto multidimensional y poseedor de diversas acepciones. Rowsell y Abrams (2011) han propuesto distinguir entre identidad e Identidad (con mayúscula), la primera referida al desarrollo psicológico, emocional y fisiológico del yo y la segunda relacionada con los procesos y prácticas con que los individuos hacen sentido y exhiben sus subjetividades en el espacio social. En este trabajo adoptamos esta segunda acepción. Entendemos la identidad no como un atributo fijo de una persona, sino en tanto fenómeno flexible y relacional (DE TEZANOS, 2012), y no como una posesión del individuo, sino una puesta en acción a través de sus prácticas en sociedad (ROWSELL; ABRAMS, 2011).

Una emergente literatura ha explorado los procesos de construcción de identidad que ocurren en la escuela, enfatizando el carácter interaccional del proceso (ESMONDE; LANGER-OSUNA, 2013) y destacando el rol de los recursos culturales que los estudiantes utilizan para reclamar ciertas identidades como propias. Williams (2011), por ejemplo, muestra cómo la cultura popular puede servir para crear referencias culturales compartidas que los escolares utilizan como significantes de sus subjetividades y mecanismos para adscribirse a comunidades más amplias.

La escuela impacta también la identidad profesional de los docentes. Su configuración comienza en su formación inicial, pero se prolonga durante todo su ejercicio profesional a través de un proceso dinámico vinculado al contexto escolar (GALAZ, 2011). Según Larrain (2001 apud DE TEZANOS, 2012), existen tres elementos que articulan la identidad profesional: 1) las categorías sociales compartidas en contextos colectivos culturalmente determinados; 2) los artefactos materiales que dan sentido de pertenencia a una comunidad deseada; y 3) la existencia de otros, que permite la construcción del sí mismo. En el caso de los docentes en Chile, su identidad se ve articulada por las categorías culturales del propio contexto escolar y las condiciones materiales de su trabajo, reflejadas tanto en los insumos de trabajo como en los sueldos percibidos (DE TEZANOS, 2012). El tercer elemento, los otros, es más difuso, pues no sólo se refiere a otros profesionales distintos a los docentes, sino también a otros profesores, y específicamente a los del propio establecimiento. Ávalos y Sotomayor (2012) plantean además que los docentes chilenos definen su identidad en referencia directa de sus alumnos, y que el reconocimiento de sus acciones depende, en gran medida, de la valoración que de ellos tengan los estudiantes, sus familias y sus colegas.

La literatura referida ilumina el rol que las prácticas cotidianas juegan en la configuración de las identidades de los actores escolares. Además, enfatiza el rol de individuos agentes que hacen sentido de sí mismos a partir de recursos culturales que forman parte de un medio social compartido y afectado por otros. Ello lleva a preguntarse por las relaciones de poder presentes en este proceso. La distinción entre identidades figurativas e identidades posicionales ilumina este aspecto. Holland y otros autores (1998) comprenden las identidades figurativas como aquellas relacionadas con "[...] las historias, actos y personajes que hacen del mundo un mundo cultural" (p.1 27). Las identidades posicionales, por su parte, estarian ligadas a las relaciones de poder interpersonales, cuya base está en la exhibición de ciertas 
identidades a través de prácticas o artefactos culturales que significan una afirmación de privilegio o posición social. Este proceso supone una relación directa entre estas prácticas y el medio social que las informa. Esmonde y Langer-Osuna (2013), en un estudio etnográfico en San Francisco, muestran cómo la capacidad de los estudiantes de exhibir sus identidades se ve directamente afectada por la interacción que éstos y sus profesores construyen al interior de la sala de clases. Cambios estructurales también pueden afectar la posicionalidad de los actores escolares, como ha ocurrido con las reformas educativas de las últimas décadas en Chile. El efecto más claro ha sido el contraste entre la celeridad con que se han producido estos cambios y el lento proceso de construcción y reconstrucción de la identidad de los docentes, lo que ha contribuido a producir rupturas y conflictos identitarios (ÁVALOS; SOTOMAYOR, 2012; DE TEZANOS, 2012).

Lo anterior apunta a la necesidad comprender cómo, en la interrelación entre estructuras sistémicas y actividades escolares, se producen las condiciones necesarias para la configuración de identidades particulares. Ello puede llegar a traducirse en valores culturales y transformarse en parte de la cultura escolar, pero también generar relaciones de exclusión que afecten a sus miembros. Convertino (2015) argumenta, para el caso norteamericano, que estos ideales culturales se manifiestan a través de microtecnologías de control que median las identidades juveniles. Estos ideales culturales y las prácticas que los sostienen se convierten en tecnologías de normalización de los individuos. Nuestro trabajo reconoce la existencia de estas microtecnologías y sus relaciones con los procesos de configuración identitaria al interior de los espacios escolares, pero se pregunta además cómo la implementación de innovaciones educativas puede abrir nuevos espacios para la configuración identitaria, dislocando algunas de estas microtecnologías y, al mismo tiempo, reforzando otras.

\section{Metodología}

Para cumplir con los objetivos de investigación realizamos un estudio de caso, analizando los efectos identitarios que ha tenido una innovación específica como es el Programa Interdisciplinario de Investigación Escolar (PIIE), un programa innovador instalado en el colegio Sagrados Corazones de Manquehue.

El colegio es un establecimiento particular pagado ubicado en el sector oriente de Santiago de Chile. Atiende a una población estudiantil proveniente del sector socioeconómico alto y tenía una matrícula de 1.901 estudiantes al año 2014. Se trata de un colegio con educación completa, que abarca desde los niveles de transición preescolar -desde los cuatro años de edad- hasta la enseñanza media o secundaria, y que funcionaba al momento de la recolección de información para este estudio bajo un modelo segregado por género -es decir, estudiantes varones y mujeres son separados en grupos-curso diferenciados, y sólo se encuentran durante recreos y actividades o asignaturas electivas-.

El PIIE nace el año 2011 como un programa extracurricular y coprogramático, es decir, fuera del currículum oficial y después del horario regular de clases, utilizando el tiempo libre de los alumnos y asignando horas extra a los profesores a cargo. Es un programa que intenta desarrollar habilidades de investigación entre los estudiantes y, a su vez, generar 
conocimiento desde la escuela. El PIIE se organiza en cuatro menciones: Historia, Lenguaje, Ciencias y Artes. Cada mención está a cargo de un docente tutor, comúnmente acompañado de uno a tres estudiantes de Cuarto Año de Enseñanza Media como ayudantes. Son estos estudiantes que, comúnmente, han participado en años anteriores del programa y que, en este nuevo rol, actúan como apoyo del tutor y nexo entre éste y los demás miembros de la mención. Finalmente, existe un docente que cumple el rol de Coordinador General del Programa, quien también es acompañado por uno o dos ayudantes.

El programa se desarrolla en ciclos anuales, siguiendo los ritmos del año escolar. Se comienza invitando a todos los estudiantes a inicio del año académico y luego los interesados se acercan a los docentes a cargo y llenan una ficha de inscripción en la cual, además de sus datos personales, deben manifestar las razones que les motivan a participar. Normalmente todos los estudiantes interesados son aceptados en el programa y, al año de realización del estudio, contaba con 62 estudiantes, lo que equivale al 12\% de la matrícula de enseñanza media del establecimiento. A inicios del mes de abril, las cuatro menciones participan de una primera reunión conjunta donde se les explica el funcionamiento del programa, para luego dar inicio a sus sesiones semanales. Las actividades se realizan conjuntamente con estudiantes de ambos géneros, los cuales se distribuyen equitativamente (33 mujeres y 29 hombres), aunque puede darse que en años específicos algunas menciones posean mayor proporción de mujeres $\mathrm{u}$ hombres, como sucedió el 2014 respectivamente con Artes e Historia.

Cada mención desarrolla un tema central cada año y de éste se desprenden diversas investigaciones que realizan parejas o grupos de estudiantes. Las sesiones semanales son el núcleo central del proceso. En ellas, los estudiantes aprenden las principales herramientas de investigación de sus disciplinas, leen y discuten bibliografía especializada, comparten con invitados externos y desarrollan, presentan y reciben comentarios sobre sus investigaciones. Hacia el final del año ocurre la transferencia del conocimiento producido a través de la presentación de algunas investigaciones en un Congreso Interdisciplinario Escolar abierto a la comunidad y por medio de la elaboración de artículos, cortometrajes, obras de arte y otros productos. Establecido el año 2011, el programa ya se ha consolidado dentro de la oferta educativa del establecimiento como un espacio coprogramático reconocido y valorado por estudiantes, docentes y apoderados.

Para recolectar la información realizamos una serie de entrevistas semiestructuradas y focus groups a diversos actores que han estado vinculados al programa, a partir de pautas confeccionadas para recoger información sobre su funcionamiento e impacto en la formación educativa de sus participantes. Se entrevistó a cuatro docentes -incluyendo al coordinador del programa-, un exdirectivo y cuatro exestudiantes, cuidando que ambos géneros estuviesen representados tanto entre los primeros como los últimos. Estas entrevistas se realizaron en sesiones de carácter individual, de una duración aproximada de una hora. Mientras que a los docentes se les entrevistó en el mismo establecimiento, al exdirectivo se le entrevistó en su lugar de trabajo actual y a los exestudiantes en cafés o en sus hogares. Además, se realizaron dos focus groups, cada uno de hora y media de duración, el primero con estudiantes de primer a tercer año de Enseñanza Media, y el segundo con estudiantes que se desempeñaban como ayudantes. Ambos grupos focales 
contaron con la misma cantidad de estudiantes hombres y mujeres y fueron realizados en una sala de reuniones del establecimiento. Dado que el programa se divide en cuatro menciones, tanto en el caso de los docentes como los estudiantes y exestudiantes se procuró tener al menos un representante de cada una de éstas, aunque en uno de los casos no fue posible por problemas de disponibilidad. En pos de resguardar su anonimato, en la sección de resultados sus voces se presentan sólo como Docentes, Estudiantes y Exestudiantes. La tabla 1 resume los informantes y métodos de recolección para cada uno.

Tabla 1- Informantes

\begin{tabular}{|c|c|c|c|}
\hline & Método & Participantes & Identificación \\
\hline Estudiantes & Focus Group & $\begin{array}{l}\text { - Estudiante Historia } \\
\text { - Estudiante Ciencias } \\
\text { - Estudiante Lenguaje } \\
\text { - Estudiante Artes }\end{array}$ & Estudiante \\
\hline Estudiantes Ayudantes & Focus Group & $\begin{array}{l}\text { - Ayudante Coordinación } \\
\text { - Ayudante Historia } \\
\text { - Ayudante Ciencias } \\
\text { - Ayudante Lenguaje } \\
\text { - Ayudante Artes }\end{array}$ & Estudiante \\
\hline Exestudiantes & Entrevistas & $\begin{array}{l}\text { - Exestudiante Ayudante Coordinación } \\
\text { - Exestudiante Historia } \\
\text { - Exestudiante Ciencias } \\
\text { - Exestudiante Arte }\end{array}$ & Exestudiante \\
\hline Docentes (Tutores) & Entrevistas & $\begin{array}{c}\text { - Coordinador (Extutor de Historia) } \\
\text { - Tutora Ciencias } \\
\text { - Tutor Lenguaje } \\
\text { - Tutora Artes }\end{array}$ & Docente \\
\hline Directivos & Entrevistas & - ExDirectivo & Docente \\
\hline
\end{tabular}

Fuente: Elaboración propia.

Cabe destacar que uno de los dos investigadores de este estudio formó parte del programa analizado en sus inicios, aunque al momento de desarrollarse la investigación ya se encontraba totalmente desligado del mismo. De todos modos, con el fin de asegurar una mayor objetividad y no comprometer los discursos de los participantes, todas las entrevistas y focus groups fueron realizados por el investigador que no tenía contacto previo con el programa.

Analizamos la información de forma inductiva, siguiendo las líneas de la Teoría Fundamentada, que destaca porque las categorías de análisis surgen a partir de los mismos datos, y no de conceptos o clasificaciones previamente definidas (GLASER; STRAUSS, 1967). Realizadas las entrevistas y focus groups, escuchamos todas las grabaciones y organizamos los reportes recogidos de acuerdo al tipo de actor emisor -Docentes o Estudiantes-. Luego, en base a una segunda escucha, construimos una serie de categorías de análisis a partir del discurso de los propios actores y volvimos a organizar la información 
a partir de éstas. Concluido esto, procedimos a identificar las relaciones existentes entre estas categorías para agruparlas en conjuntos mayores, proceso que realizamos dos veces en pos de establecer los ejes y sub-ejes temáticos que estructuran este artículo, según se muestra en la Tabla 2. De esta forma fuimos capaces de identificar y examinar con mayor claridad las distintas dimensiones del proceso de producción de identidades que forma parte de la innovación educativa estudiada.

Tabla 2- Ejes temáticos y categorías de análisis

\begin{tabular}{|c|c|c|}
\hline Categorías de análisis & Sub-ejes temáticos & Ejes temáticos \\
\hline $\begin{array}{c}\text { "Ir más allá de la sala de clases" } \\
\text { "Ser distinto" } \\
\text { "Alumno multifacético" } \\
\text { "Profesores jóvenes" }\end{array}$ & Discursos de Identidad & \multirow{3}{*}{$\begin{array}{l}\text { NuEVO ESPACIO DE PRODUCCIÓN DE } \\
\text { IDENTIDADES }\end{array}$} \\
\hline $\begin{array}{l}\text { Prácticas intencionadas } \\
\text { Prácticas no intencionadas }\end{array}$ & $\begin{array}{l}\text { Prácticas de configuración de } \\
\text { identidad }\end{array}$ & \\
\hline $\begin{array}{l}\text { Búsqueda de reconocimiento } \\
\text { Ganancia de espacios físicos y/o simbólicos }\end{array}$ & Reconocimiento exterior & \\
\hline $\begin{array}{l}\text { Autoconocimiento como aprendizaje } \\
\text { Autoconocimiento como orientación }\end{array}$ & Espacio de autoconocimiento & \multirow{3}{*}{$\begin{array}{l}\text { IMPLICANCIAS DE NUEVAS IDENTIDADES } \\
\text { PRODUCIDAS }\end{array}$} \\
\hline $\begin{array}{l}\text { Sentido de pertenencia } \\
\text { Sentido de apropiación }\end{array}$ & Sentido de comunidad & \\
\hline $\begin{array}{l}\text { Refuerza nociones previas de elite escolar } \\
\text { Construye nuevas nociones de elite escolar }\end{array}$ & Peligro de elitización & \\
\hline
\end{tabular}

Fuente: Elaboración propia.

\section{Resultados}

\section{La construcción de un nuevo espacio de identidad}

En las entrevistas identificamos una serie de elementos que han ido configurando en el establecimiento un nuevo espacio de identidad. El primero tiene que ver con los discursos compartidos que los actores han construido en relación al PIIE y a través de los que perciben a quienes participan de éste.

Un primer discurso tiene relación con el PIIE en tanto espacio que amplía la mirada educativa tradicional. Los informantes concuerdan en que quienes participan de este programa son individuos interesados en ir "más allá de la sala de clases". El discurso asume que el aula regular tiene limitaciones propias -con expresiones como que la sala "queda chica" y que tiene un "techo" que muchos estudiantes ya alcanzaron- que impiden profundizar en las distintas disciplinas que forman parte del currículum oficial. PIIE lograría romper esas limitaciones a través de la especialización de los alumnos en alguna de estas disciplinas por medio de la investigación. Los docentes concuerdan en que muchas de estas limitaciones son causadas por un sistema sobrerregulado por las 
evaluaciones estandarizadas, un currículum extenso y un modelo de accountability que alienta la búsqueda de resultados concretos por sobre los procesos de aprendizaje. Así, se posicionan buscando superar los límites que el sistema escolar tradicional les impone, alineándose con los estudiantes del PIIE en este ir "más allá de la sala de clases".

Un segundo discurso se relaciona directamente con los estudiantes del PIIE, a quienes se asocia con un perfil multifacético. Se les identifica como alumnos que participan de otras actividades extraprogramáticas, como deportes, cultura, pastoral y acción social o Centro de Estudiantes. Los informantes plantean que, por lo tanto, son alumnos con inquietudes, y comprometidos con el colegio. Los estudiantes además señalan que este carácter multifacético es lo que les permite estar en PIIE, pues son capaces de organizar bien su tiempo y sus deberes.

Un tercer discurso diferencia además a estos estudiantes de sus compañeros. Perciben que el resto de la comunidad escolar los considera diferentes -como "raritos", "ñoños", "bohemios" o "hipsters"-. A pesar de que algunos de estos adjetivos son peyorativos, ellos se sienten orgullosos de esta identificación, pues la asocian a su pasión por aprender. Sin embargo, esta distinción revela también cómo se define la normalidad entre los estudiantes del establecimiento, y cómo su participación en este espacio es necesariamente disruptiva respecto a ésta. Cabe destacar que este discurso pertenece casi exclusivamente a los estudiantes, y sólo un docente hace referencia a esta temática.

Los últimos dos discursos son propios de los docentes. El primero se refiere al perfil de quienes asumen la labor de tutores, que es de profesores con energía, ganas de innovar y jóvenes. Juventud aquí es una categoría no tanto etárea sino ontogenética: el discurso habla de profesores que por no tener responsabilidades familiares pueden dedicar más tiempo a esta labor, y cuya motivación es alimentada por ideas novedosas traídas de su reciente formación universitaria. Este discurso, sin embargo, se muestra también desde el riesgo, pues se reporta la juventud como una condición necesaria para pertenecer al proyecto, mientras se admite la dificultad de mantenerse trabajando en el programa cuando comienzan a aparecer en la vida responsabilidades familiares o mayores cargas horarias.

Junto a lo anterior, un último discurso asocia cierto heroísmo con aquellos profesores que participan del proyecto. Así, se identifican como profesores que van más allá de su trabajo aunque la recompensa monetaria o el reconocimiento no se corresponda. PIIE se convierte en un sacrificio o un deber ético, que toman con la motivación necesaria para potenciar los aprendizajes de sus alumnos y a la vez resolver inquietudes propias de su desarrollo profesional. Esta motivación se ve como una condición necesaria para la participación en el proyecto -incluso admitiendo que este no funcionaría si los profesores estuvieran ahí únicamente por mandato o incentivos materiales- aunque se complementa con una mirada crítica, que busca una retribución justa a la labor realizada, coherente con la importancia que estos docentes conceden al programa.

Junto a estos discursos, un segundo elemento referido a la construcción de este nuevo espacio de identidad tiene que ver con las prácticas a través de las cuales se configura. Las entrevistas revelan un progresivo interés en promover una identidad PIIE tanto entre docentes como estudiantes. Los exestudiantes rememoran que durante sus primeros años en el PIIE sentían que había pocos espacios de encuentro con los miembros de otras menciones, lo que llevó a la generación de instancias explícitamente diseñadas 
para alcanzar este objetivo, como asados o una jornada de cierre de año donde todas las menciones visitaron Valparaíso. De acuerdo a los estudiantes menores, estas prácticas intencionadas han sido efectivas, ayudándolos a descubrir lo que comparten con aquellos participantes de otras menciones y el interés común que poseen en que el Programa funcione y continúe:

Y, como que ahí como que te vai dando cuenta que en realidad tenemos más cosas en común que diferencias. 0 sea como que la única diferencia es qué te gusta y en qué te queris especializar, pero todos tenemos como... la inquietud de querer saber más y, y, eso como de que, como de que te sentís más comprometido con, no sé si con lo académico pero por, por, como por generar conocimiento. Y como que, cuando nos juntamos entre todos, de repente salen conversaciones muy interesantes entre personas como de distintas menciones y con los profesores, cuestiones así. (Estudiante, Santiago, 04-09-14).

Los entrevistados también reportan la existencia de una serie de prácticas que, sin tener como objetivo central el promover un nuevo espacio identitario, contribuyen de forma importante a ello. Una de éstas es el trabajo en equipo, reconocida por estudiantes y docentes como una de las principales características pedagógicas del programa. La mención de Artes es el ejemplo más claro de esto: el año 2013, sus alumnos se conformaron como un solo gran colectivo. Los estudiantes del PIIE perciben que en este trabajo en equipo se va construyendo un sentido de colectividad, diferenciándolo de aquel promovido al interior de la sala de clases, donde la preocupación por la calificación obstaculiza una verdadera colaboración y aprendizaje mutuo. A esto se suman otras prácticas relacionadas al proceso de investigación y la transferencia del conocimiento producido -como las salidas a terreno con el fin de recolectar datos para sus investigaciones, o el Congreso Interdisciplinario Escolar- que los actores reconocen como importantes en el establecimiento y fortalecimiento de lazos al interior y entre las distintas menciones.

En contraposición, observamos que no existen prácticas -intencionadas o no- que promuevan una identidad PIIE entre los docentes. Esto puede atribuirse a la escasez de tiempo y falta de espacios destinados al trabajo conjunto, que impide que los profesores tutores se encuentren y se identifiquen como colectivo docente.

Este ensamblaje de prácticas contribuye a la producción de un sentido de comunidad. Las actividades gregarias son enormemente importantes en esto, pues los actores reconocen que es en el compartir donde generan lazos entre ellos. A la vez, se establece una relación bidireccional entre estos lazos y los procesos de investigación que los estudiantes desarrollan. Es a través de la cooperación y el trabajo conjunto que los lazos se forman, pero estos lazos son, a la vez, una herramienta que contribuye a producir investigaciones de mayor calidad y más conectadas entre sí. Ello, sumado a la posición que los docentes asumen en el programa - en tanto facilitadores de herramientas, y no exclusivamente proveedores de conocimientos- y la labor que cumplen los ayudantes en tanto intermediarios, produciría lo que, en palabras de uno de los docentes, puede definirse como "un crecimiento en comunidad".

Un último elemento referido a la configuración de este espacio de identidad se relaciona con el reconocimiento externo que recibe el programa. Uno de los docentes 
recalca constantemente en su narrativa la importancia de hacerse notar en el colegio y ganar espacios simbólicos. La comparación obligada es con el área de Educación Física -que en este establecimiento en particular tiene un espacio importante ya ganado-, la que es visible a través de copas, campeonatos, invitaciones a la comunidad a alentar a las selecciones, e incluso al tener estudiantes entrenando en los patios. Las actividades de PIIE servirían así para ganar espacios tanto materiales como simbólicos -publicaciones, ferias de ciencias, reconocimiento público-, lo que contribuiría a una mayor identificación tanto de los estudiantes como de los docentes con el proyecto.

Al respecto, la evaluación de los actores es que el PIIE ha ido ganando con el tiempo una imagen positiva al interior de la comunidad escolar. En sus relatos reconocen que al principio dicha imagen prácticamente no existía, pero que fue forjándose cuando comenzaron a realizarse prácticas intencionadas, como la presentación de investigaciones a sus compañeros en aulas de clases, los congresos anuales o salidas a terreno abiertas al resto de la comunidad escolar. El ejemplo más notorio a la fecha del estudio, sin embargo, es un gran mural desarrollado por la mención de Artes, que cubre toda una muralla dentro del colegio y es visible para toda la comunidad. Como señala uno de los docentes:

El PIIE se está haciendo presente de a poco. A inicio de año, más allá del congreso de inicio de año, el asado. Se hizo un asado entre los chiquillos para mirarse. El de Ciencias que conozca al de Lenguaje, y se van conociendo. El año pasado salimos a Valparaíso. Todo el PIIE salió a Valparaíso un Día del Patrimonio, o sea, una salida patrimonial en diciembre. El Día del Patrimonio ya ha sido ya tres años seguidos como una salida desde el PIIE que invita a todo el colegio. Entonces el PIIE invita al colegio, y esto sale en la página, entonces se va ganando un espacio a nivel de lenguaje. Que la presidenta del año pasado y la presidenta de este año del Centro de Alumnos mencione como meta, las cosas buenas que tiene este colegio, y mencione el PIIE en un acto cívico, es porque nos estamos ganando un espacio, estamos en el discurso. (Docente, Santiago, 04-09-14).

En síntesis, identificamos tres elementos en el PIIE que aportarían a la construcción de un nuevo espacio de identidad en este establecimiento. En primer lugar, una serie de discursos relacionados tanto a los estudiantes como a los docentes, sobre sus propias características individuales y colectivas. Segundo, un conjunto de prácticas concretas, algunas intencionadas para configurar una identidad propia, y otras que no tienen dicho fin pero que contribuyen a ello. Por último, el reconocimiento externo que provee el resto de la comunidad escolar y que se convierte tanto en objetivo como en recurso para dicha configuración identitaria.

\section{Implicancias de la construcción de un nuevo espacio de identidad}

A través de las entrevistas, estudiantes y docentes no sólo dan cuenta del proceso de formación de un nuevo espacio de identidad, sino también de lo que éste ha significado para ellos, tanto a nivel individual como colectivo. Por medio de sus relatos, es posible observar algunas de las formas en que conceptualizan y presentan el impacto que este programa ha tenido sobre sus identidades. 
Desde el punto de vista del impacto sobre las identidades individuales de los estudiantes, observamos dos efectos consistentemente reportados. El primero se refiere al autoconocimiento personal. Los estudiantes reportan diversas razones para ingresar al PIIE, pero la más recurrente es el interés por las temáticas de cada mención. Sin embargo, al momento de referirse a los resultados del proceso, no es el aprendizaje sobre las temáticas lo que destacan, sino más bien el impacto que su participación ha tenido en cómo se observan a sí mismos. Los aprendizajes al respecto son diversos y van desde aprender a qué hora prefieren leer o qué técnicas les ayudan a concentrarse más, hasta el desarrollar la capacidad crítica respecto a sus propios productos. Sin duda uno de los más importantes entre estos aprendizajes es el aprender a valorarse. Para los estudiantes, el PIIE es una instancia que les ha permitido "creerse el cuento", "empoderarse", superar la timidez y los miedos e incluso "callar bocas" cuando han sentido que sus pares, otros docentes e incluso ellos mismos no han creído en lo que pueden lograr. Los docentes del programa concuerdan con esta percepción, destacando su efecto en la personalidad y autoestima de sus miembros.

El segundo efecto reportado transversalmente se refiere al impacto del programa en los procesos de orientación vocacional de los estudiantes. El programa es visto como un espacio que les conecta con la realidad del mundo universitario e incluso profesional, lo que es confirmado fervientemente por aquellos exestudiantes que han seguido caminos de formación afines a alguna de las distintas menciones:

Como que te saca un poco el mito de la cuestión [...]. Entonces no sé po, yo por ejemplo hoy en día igual quiero hacer la pedagogía y me gustaría ser profe, pero siempre en paralelo con la investigación, como que ya es un camino que no quiero dejar nunca ¿cachai? Y yo creo que sin el PIIE como que... como que no sé si es que me haya dado la decisión, pero en el fondo me dio una noción real de lo que era. (Exestudiante, Santiago, 30-08-14).

Además del conocimiento experiencial que el PIIE entrega respecto a las disciplinas que lo componen, sus miembros reconocen en éste una instancia donde los intereses vocacionales se ponen a prueba. En palabras de uno de los docentes, PIIE es un espacio "[...] de autoexploración académica de los chiquillos, de probar" (Docente, Santiago, 0409-14). La mayoría de los estudiantes concuerda con ello, reconociendo que en el programa han podido descubrir si quieren dedicar sus futuros profesionales a sus áreas de interés o no. Incluso en los casos donde la respuesta final ha sido negativa, los estudiantes no lo perciben como un fracaso o una razón para alejarse del programa, sino que lo valoran en tanto proceso de aprendizaje sobre sí mismos, el cual les entrega herramientas para definir de mejor manera su futuro:

[...] me ha quitado un poco el miedo [...] tengo muchas compañeras que están demasiado estresadas porque 'ay, no sé qué quiero estudiar y no sé qué.' El PIIE me ha dado las herramientas para sentirme segura de la inseguridad y como de poder dar un giro en 180 grados y decir como 'no, yo no quiero hacer esto y quiero hacer esta otra cosa'. (Estudiante, Santiago, 04-09-14). 
Desde el punto de vista del impacto del programa en las identidades individuales de los docentes, observamos que éste se relaciona casi exclusivamente con su desarrollo profesional. Según sus relatos, el programa funciona para ellos como un espacio de aprendizaje, en tanto les provoca nuevas preguntas e inquietudes e incluso los lleva a modificar sus comportamientos en el aula de clases regular. Reconociendo que el establecimiento les ha brindado oportunidades para desarrollarse en tanto docentes, y que esto se refleja en ésta y otras innovaciones educativas de las que participan, los docentes valoran que el programa les permita ganarse un espacio al interior de la comunidad escolar.

Además de un impacto en las identidades individuales, observamos que existe un impacto del programa a nivel colectivo, en la construcción de un fuerte sentido de comunidad. Éste puede definirse a partir de dos dimensiones.

En primer lugar, existe un fuerte sentido de pertenencia de parte de los estudiantes con respecto al programa. Ellos refieren que el PIIE les gusta no sólo por el trabajo que realizan, sino por el ambiente que se genera entre sus miembros. Lo consideran un espacio en que encuentran temas, motivaciones y un lenguaje en común, lo que abre instancias para compartir y conversar con sus pares. Estas relaciones destacan además porque rompen las barreras propias de la estructura de niveles y cursos del colegio, teniendo carácter intergeneracional e intergénero. Además, se le diferencia de otras actividades extraprogramáticas que ya existían -como las selecciones deportivas o el grupo de teatro-, planteándose como un espacio para los estudiantes que no tenían un lugar donde explorar este tipo de intereses.

Junto con este sentido de pertenencia se genera también un sentido de apropiación. Los estudiantes no sólo perciben que encontraron un lugar en que se sienten cómodos, sino que además plantean que les pertenece y lo sienten suyo, teniendo un fuerte impacto en sus biografías, lo que a su vez generaría una mayor vinculación afectiva con el programa. Este sentido de apropiación se percibe principalmente en dos aspectos. Uno es el orgullo que generan en los estudiantes los productos de sus investigaciones, particularmente cuando éstas son publicadas o reconocidas por otros. Más de un estudiante reportó este sentimiento al haber sido citado en trabajos realizados por personas internas y externas al establecimiento, remarcando que su investigación poseía valor para otros, además de ellos mismos y sus tutores. Un segundo aspecto es la inquietud que varios de sus miembros sienten por retribuir al programa, particularmente participando de éste como ayudantes. Esto refuerza su convicción de que éste no es un programa sólo para los estudiantes, sino uno que "se hace entre todos":

La dinámica del PIIE de Arte es como muy, muy libre, como [la tutora] nos dice que, que en el fondo nosotros tenemos que guiar el PIIE, que el PIIE lo hacemos nosotros, es más como desde nosotros hacia ella casi, que de ella hacia nosotros. (Estudiante, Santiago, 04-09-14).

Este sentido de comunidad, sin embargo, no se ha construido de la misma forma entre los docentes. Como se mencionó anteriormente, el trabajo se da entre ellos de forma parcelada, lo que impide una identificación como colectivo con el programa. Sin embargo, 
algunas señales indican que sí se construye en los docentes una identificación con el PIIE a través de su relación con los alumnos, lo que se infiere de algunas ideas expresadas por estudiantes y docentes respecto a la interacción más cercana que en este contexto existe entre ambos actores:

Como que igual el profe siempre representa una cierta imagen de autoridad, a pesar de que los profes del PIIE trataban de romper un poco con eso, como de generar una relación un poco más cercana. (Exestudiante, Santiago, 30-08-14).

A esto se suma que los docentes utilizan en variadas oportunidades la primera persona plural al hablar del trabajo que se realiza en el PIIE, difuminando la distinción entre docentes y estudiantes y reforzando la hipótesis de que es en su relación con éstos donde construyen su identificación con el programa.

El impacto del programa sobre las identidades de sus miembros ha implicado, sin embargo, un riesgo de elitización de este nuevo espacio de identidad. Ésta es una preocupación que reportan principalmente algunos docentes y aquellos exalumnos que formaron parte del PIIE durante su primer año, cuando sólo ciertos estudiantes eran invitados a participar. El proceso de ingreso se cambió al año siguiente por una invitación abierta, lo que aminoró el riesgo de que sus miembros fueran vistos como una élite escolar, aunque ciertamente no acabó con éste. Los estudiantes reportan que quienes no participan del programa los tildan de ser "los mejores alumnos" o de formar parte de una "secta". Sin embargo, también reconocen percibir un trato diferencial por parte de los profesores de aula que no son tutores del programa, quienes modifican positivamente la opinión que tienen sobre ellos una vez que saben que forman parte del PIIE.

El peligro reside no sólo en la elitización con relación al resto de la comunidad escolar, sino además en que ésta puede provocar prácticas de exclusión que atenten contra el mismo espacio que el programa ha abierto, obstaculizando el ingreso de estudiantes que no se sientan capaces de cumplir con los estándares que perciben desde su exterior:

[...] a mí me ha tocado conversar con muchas niñas como que dicen de repente 'a mí me hubiese gustado meterme al PIIE pero como que no me hubiesen aceptado' y es como, ves, 'aceptan a toda la gente', no sé por qué, 'no, pero es que se meten solo los inteligentes’ y es como: ‘No!' ¿Cachai? Como que existe mucho esa idea como de que solamente los que les va bien o como los más inteligentes, qué se yo, se meten. (Estudiante, Santiago, 04-09-14).

Estas barreras incluso pueden aparecer al interior del programa, como refleja el caso de una estudiante que, al momento de escoger en qué mención participar, reportó haber descartado una de ellas por no sentirse "capaz de hacer esos trabajos tan buenos como los que han hecho” (Estudiante, Santiago, 04-09-14). En casos como éste, el PIIE deja de verse como un nuevo espacio de identidad y pasa a ser percibido como una identidad en sí misma -la de élite escolar- que se entiende como requisito de ingreso y membresía del programa, con todos los procesos de exclusión que ello conlleva. 


\section{Conclusiones}

Toda innovación implica cambios y las innovaciones educativas no son la excepción. Pero una concepción meramente técnica impide entender de qué forma estos cambios involucran siempre a una comunidad que transforma sus prácticas, creencias y valores, produciendo muchas veces impactos inesperados. Hemos examinado este proceso a través del análisis del PIIE, una innovación educativa que busca posicionar la investigación como una práctica relevante al interior de la escuela, pero cuya implementación ha abierto además un nuevo espacio de identidad. De esta forma, el PIIE ejemplifica cómo una innovación educativa interactúa con los miembros de un establecimiento y su cultura escolar específica. También, cómo en estas interacciones encontramos aprendizajes y productos enormemente relevantes para nuestras escuelas y que una mirada instrumental dejaría escondidos.

El objetivo del PIIE es la formación de estudiantes en tanto investigadores. Ello no sólo supone que la investigación está al centro de esta innovación educativa sino además que entrega a los estudiantes recursos y orientaciones hacia el futuro, estructura sus prácticas y se establece como polo que atrae a pares con similares inquietudes. Al mismo tiempo, la investigación es reconocida como objetivo ideal, pero no presente en el espacio escolar cotidiano y, desde esta perspectiva, sustenta el discurso identitario central de los miembros del programa: aquel referido a ir "más allá de la sala de clases". Esto tiene dos implicancias fundamentales. En primer lugar, permite a docentes y estudiantes producir nuevas identidades figurativas con las que establecer un mayor sentido de comunidad a la vez que diferenciarse de sus pares que no participan del programa. En segundo lugar, contribuye a establecer al programa y a sus miembros en oposición a las estructuras sistémicas del establecimiento y del sistema escolar chileno en general.

Este último punto es de particular relevancia, en tanto impacta prácticas escolares que se establecen como recursos culturales para la producción de identidades y que a la vez cobran nuevo sentido pedagógico. El trabajo en equipo es aquí el ejemplo por excelencia. La transformación que ocurre con esta práctica al interior del PIIE no es producto de un cambio de tipo pedagógico o didáctico, sino más bien resultado de un proceso de producción identitaria de carácter simbólico, pero también material. El trabajo en equipo al interior del PIIE no es distinto al del aula por cómo se hace, sino por quiénes lo hacen -estudiantes investigadores, que comparten un interés común- y el motivo por el que se hace -no por una calificación personal, sino un producto colectivo, que permite seguir construyendo el programa en su conjunto-. Si bien comparar el trabajo en equipo al interior del aula de clases regular y aquel PIIE requeriría un estudio de carácter etnográfico, los reportes de los miembros del programa revelan que ellos mismos han realizado esta comparación y comprendido que el sentido del trabajo en equipo va mucho más allá de los resultados del mismo en términos de calificaciones, un aprendizaje esencial que, para muchos docentes, es un desafío a lograr en el aula regular. Nuestra investigación sugiere que alcanzar este objetivo requiere no sólo enfocarse en sus elementos didácticos, sino en la relación que se establece entre éste y los procesos de producción de identidades que los estudiantes se encuentran viviendo. 
El PIIE se establece así como un espacio de producción de identidades en práctica. No podemos enfatizar lo suficiente la dimensión productiva del proceso, pues el riesgo es pensar que el PIIE ha permitido a cierto grupo de estudiantes manifestar una identidad que ya poseían y que hasta entonces no tenía vías de expresión. Nuestra evidencia muestra un proceso distinto, donde el reconocimiento de los estudiantes en tanto multifacéticos niega la existencia de una identidad única y reprimida hasta entonces. Las narrativas de los actores sobre las prácticas de configuración de identidad del programa y su desarrollo en el tiempo muestran además cómo identidad y práctica están íntimamente ligadas en este proceso. Este programa se configura así como un nuevo espacio de práctica que permite a estudiantes y docentes producir nuevas identidades figurativas para sí mismos.

A su vez, el PIIE es también un espacio de producción y reconfiguración de identidades posicionales. En este aspecto, las implicancias de la innovación pueden ser contradictorias. Las nuevas identidades producidas en este espacio son disruptoras de lo que se percibe como la normalidad escolar y, desde este punto de vista, pueden convertirse en herramientas para que algunos estudiantes escapen de espacios de exclusión, logren forjar un sentido de pertenencia, y encuentren reconocimiento externo por parte de sus pares y la comunidad educativa. Pero al mismo tiempo, los procesos de producción de estas nuevas identidades pueden contribuir a lógicas de exclusión asociadas a criterios de excelencia académica, reforzando procesos de elitización ya existentes o incluso estableciendo una nueva élite escolar. En este segundo escenario, la participación en el PIIE no proporciona herramientas para ningún tipo de liberación, sino que la membresía en el mismo se convierte en una práctica de exclusión. Ello supone asumir que una innovación como ésta existe siempre en un delicado balance entre contribuir a romper con las microtecnologías de normalización propias del espacio escolar y convertirse en una de éstas. Los actores que participan de esta innovación son responsables de este balance y es importante que se hagan cargo del mismo de modo explícito.

Estos procesos de producción identitaria se desarrollan en el contexto del PIIE en tanto innovación educativa. Comprender los múltiples impactos que innovaciones como estas pueden tener requiere entonces entenderlas en al menos dos niveles. Por un lado, desde su implementación, que proporciona recursos y escenarios que no existían previamente al interior del establecimiento y que permiten la producción de nuevas prácticas y, por medio de estas, de nuevas identidades. Por el otro, desde la participación en la innovación, a través de la cual nuevos recursos y artefactos culturales son creados y apropiados por los actores. En tanto toda identidad no es una posesión del individuo sino una dimensión necesariamente relacional, su producción nunca es resultado de un proceso mecánico y automático. No es la mera existencia de una innovación y el acceso de estudiantes y docentes a ésta lo que produce nuevas identidades, sino que son las prácticas sociales que la innovación permite las que construyen un nuevo espacio identitario. Es en el proceso de participar de este espacio y hacerlo propio que los actores involucrados están constantemente produciendo sus identidades. Si todo aprendizaje es siempre un proceso de transformación, entonces entender las relaciones entre innovaciones educativas y producción de identidades en nuestras escuelas no es sólo un objetivo deseable. Es necesario. 


\section{Referencias}

ALTOPIEDI, Mariana; LÓPEZ JIMÉNEZ, Carlos. Contextos difíciles e historias turbulentas como motores de la innovación. Profesorado, Granada, v. 14, n. 1, p. 29-45, 2010.

ANDERSON, Theresa. The 4Ps of innovation culture: conceptions of creatively engaging with information. Information Research, Borås, v. 18, n. 3, p. 1-16, 2013.

ASTUDILLO, Gustavo; IMBARACK, Patricia. El sentido del mejoramiento escolar de los docentes en el contexto de políticas de apoyo externo. Pensamiento Educativo, Santiago de Chile, v. 50, n. 2, p. 132 146, 2013.

ÁVALOS, Beatrice; SOTOMAYOR, Carmen. Cómo ven su identidad los docentes chilenos. Perspectiva Educacional, Viña del Mar, v. 51, n. 1, p. 77-95, 2012.

BOURDIEU, Pierre; PASSERON, Jean-Claude. La Reproducción. Elementos para una teoría del sistema de enseñanza. México D.F.: Fontamara, 2005.

CONVERTINO, Christina. Misfits and the imagined American high school: a spatial analysis of student identities and schooling. Anthropology y Education Quarterly, Amherst, v. 46, n. 4, p. 363-379, 2015.

DE TEZANOS, Araceli. ¿ldentidad y/0 tradición docente? Apuntes para una discusión. Perspectiva Educacional, Viña del Mar, v. 51, n. 1, p. 1-28, 2012.

ESMONDE, Indigo; LANGER-OSUNA, Jennifer. Power in numbers: student participation in mathematical discussions in heterogeneus spaces. Journal for Research in Mathematics Education, Reston, v. 44, n. 1, p. 288-215, 2013.

FULLAN, Michael. The new meaning of educational change. 4. ed. v. 84. New York: Routledge, 2007.

GAIRÍN, Joaquín; RODRÍGUEZ-GÓMEZ, David. Cambio y mejora en las organizaciones educativas. Educar, Barcelona, v. 47, n. 1, p. 31-50, 2011.

GALAZ, Alberto. El profesor y su identidad profesional. ¿facilitadores u obstáculos del cambio educativo? Estudios Pedagógicos, Valdivia, v. 37, n. 2, p. 89-107, 2011.

GARCÍA, Rodrigo. Innovación, cultura y poder en las instituciones educativas: la complejidad en el cambio de educación. Educar, Barcelona, n. 35, p. 11-27, 2005.

GLASER, Barney; STRAUSS, Anselm. The discovery of grounded theory: strategies for qualitative research. Chicago: Aldine, 1967.

HOLLAND, Dorothy et al. Identity and agency in cultural worlds. Cambridge: Harvard University Press, 1998. 
JONNAERT, Philippe et al. La competencia como organizadora de los programas de formación: hacia un desempeño competente. Profesorado, Granada, v. 12, n. 3, p. 1-32, 2008.

KEZAR, Adrianna. Understanding and facilitating organizational change in the 21st century. ASHE-ERIC higher education report. v. 28. San Francisco: Jossey-Bass, 2001.

LOPES, Amelia. La construcción de identidades docentes como constructo de estructura y dinámica sistémicas: Argumentación y virtualidades teóricas y prácticas. Profesorado. Revista de currículum y formación del profesorado, Granada, v. 11, n. 3, p. 1-25, 2008.

MENCHÉN, Francisco. Atrévete a ser creativo. Pasos para ser creativos. Reice, Madrid, v. 10, n. 2, p. 248263, 2012.

MONTERO, Lourdes; GEWERC, Adriana. De la innovación deseada a la innovación posible: escuelas alteradas por las TIC. Profesorado, Granada, v. 14, n. 1, p. 303-318, 2010.

MURILLO, Javier. La «mejora de la escuela»: Concepto y caracterización. In: MURILLO, Javier; MUÑOZREPISO, Mercedes (Ed.). La mejora de la escuela. Barcelona: Octaedro, 2002. p. 15-51.

REYES JUÁREZ, Alejandro. La escuela secundaria como espacio de construcción de identidades juveniles. Revista Mexicana de Investigación Educativa, Ciudad de México, v. 14, n. 40, p. 147-174, 2009.

RIVAS, Manuel. Innovación educativa: teoría, procesos y estrategias. Madrid: Síntesis, 2010.

ROWSELL, Jennifer; ABRAMS, Sandra Schamroth. (Re)conceptualizing I/identity: an introduction. Teachers College Record, New York, v. 113, n. 13, p. 1-16, 2011.

SAHLBERG, Pasi; BOCE, Elona. Are teachers teaching for a knowledge society? Teachers and Teaching, Nottingham, v. 16, n. 1, p. 31-48, 2010.

SEPÚLVEDA, Carmen; MURILLO, Javier. El origen de los procesos de mejora de la escuela: un estudio cualitativo en 5 escuelas chilenas. Reice, Madrid, v. 10, n. 3, p. 6-24, 2012.

WILLIAMS, Bronwyn. Collages of identity: popular culture, emotion and online literacies. Teachers College Record, New York, v. 113, n. 13, p. 200-219, 2011.

Recibido en: 12.04 .2018

Revisiones en: 25.09.2018

Aprobado en: 29.10.2018

Rodrigo Mayorga es magíster en Historia y candidato a doctor en Antropología Educacional por el Teachers College de Columbia University. Sus intereses de investigación abarcan la educación ciudadana, el activismo estudiantil y la conciencia histórica en escuelas secundarias. 
Javier Pascual es sociólogo, máster en Investigación en Educación y doctorando en Educación por la Universitat Autònoma de Barcelona. Actualmente es Director de Estudios y Consultorías en focoSocial. Ha desarrollado diversos estudios sobre innovación educativa, liderazgo pedagógico, evaluación de aprendizajes y política educativa. 